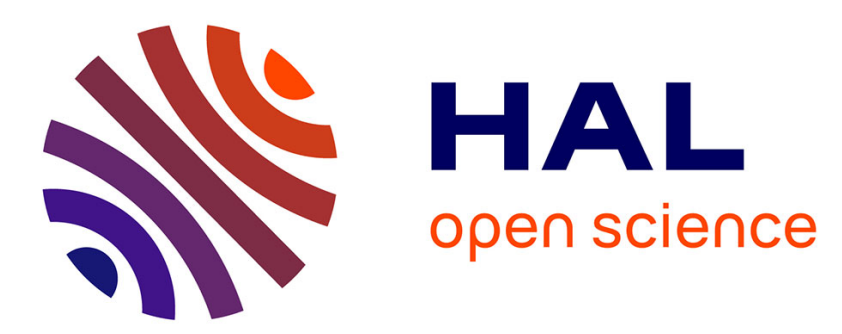

\title{
Numerical Simulation of Spin Coating Processes involving Functionalised Carbon Nanotube Suspensions
}

Elías Cueto, Anson W K Ma, Francisco Chinesta, Malcolm Mackley

\section{To cite this version:}

Elías Cueto, Anson W K Ma, Francisco Chinesta, Malcolm Mackley. Numerical Simulation of Spin Coating Processes involving Functionalised Carbon Nanotube Suspensions. International Journal of Material Forming, 2008, 1 (2), 10.1007/s12289-008-0377-5 . hal-00287285

\section{HAL Id: hal-00287285 \\ https://hal.science/hal-00287285}

Submitted on 15 Mar 2018

HAL is a multi-disciplinary open access archive for the deposit and dissemination of scientific research documents, whether they are published or not. The documents may come from teaching and research institutions in France or abroad, or from public or private research centers.
L'archive ouverte pluridisciplinaire HAL, est destinée au dépôt et à la diffusion de documents scientifiques de niveau recherche, publiés ou non, émanant des établissements d'enseignement et de recherche français ou étrangers, des laboratoires publics ou privés. 


\title{
Numerical simulation of spin coating processes involving functionalised Carbon nanotube suspensions
}

\author{
E. Cueto • A. W. K. Ma • F. Chinesta - M. \\ R. Mackley
}

\begin{abstract}
This paper reports the numerical simulation of spin coating for functionalised Carbon Nanotube (CNT) suspensions. Spin coating is a process commonly used to deposit uniform thin films onto flat substrates by means of high rotation velocity and centrifugal force. The functionalised CNTs modelled in this study were chemically treated in a way such that aggregation was prevented through electrostatic repulsion between CNTs. The functionalised CNTs in the semi-dilute suspensions can be modelled as rigid fibres with their orientation dictated by the flow of the solvent. The evolution of CNT orientations was simulated using a pre-averaged kinetic theory with an appropriate rotary diffusion coefficient accounting for randomising events. A Natural Element (NE) strategy with an updated Lagrangian framework was implemented to solve the free-surface problem involving large domain deformation and to avoid numerical problems associated with Finite Element (FE) modelling. The model reported herein couples micro-scale CNT orientation with the macroscopic suspension kinematics and it offers important insights in relation to the final properties of spin-coated CNT films as well as the processing behaviour of CNT suspensions.
\end{abstract}

Keywords Carbon nanotubes $\cdot$ Spin coating $\cdot$ Numerical simulation $\cdot$ Meshless

Work partially supported by the Spanish Ministry of Education and Science through grant number CICYT-DPI2005-08727-C02-01.

E. Cueto

Group of Structural Mechanics and Material Modelling. Aragón Institute of Engineering Research, I3A. Universidad de Zaragoza. Edificio Betancourt. María de Luna, 7. E-50012 Zaragoza, Spain. URL: http://gemm.unizar.es. E-mail: ecueto@unizar.es

A. Ma and M. R. Mackley

Department of Chemical Engineering, U. of Cambridge. New Museums Site, Pembroke Street. Cambridge, CB2 3RA, U.K. URL: http://www.cheng.cam.ac.uk/research/groups/polymer/ E-mail: mrm5@cam.ac.uk

F. Chinesta

Laboratoire de Mecanique des Systemes et des Procedes. UMR 8106 CNRS-ENSAMESEM. Paris, France. URL: http://www.paris.ensam.fr/lmsp/ E-mail: francisco.chinesta@paris.ensam.fr 


\section{Introduction}

Carbon nanotubes have generated a tremendous interest in the scientific community following a journal publication by Iijima in 1991 [15]. Single-walled carbon nanotubes (SWNT) are one-atom thick sheets of graphite (called graphene) rolled up into seamless cylinders with diameter in the order of a nanometer. They posses impressive mechanical properties:

- Young's modulus: 1 to $5 \mathrm{TPa}$

- Tensile Strength: 13-53 GPa

- Elastic strain up to $5 \%$.

- Density $2160 \mathrm{~kg} / \mathrm{m}^{3}$.

Given these properties, CNTs have been extensively researched as possible reinforcement (and conductive elements) for high-performance nanocomposites. In addition, nanotube based transistors have been made that operate at room temperature and that are capable of digital switching using a single electron [20].

In order to design manufacturing processes more effectively, it is important to understand and model the behaviour of CNT suspensions and the orientation of CNTs. Although several studies have focused on producing polymer nanotube composites, many practical challenges remain before their potential can be fully realized. Dispersing the nanotubes individually and uniformly into the matrix seems to be fundamental in producing composites with reproducible and optimal properties.

This work is aimed at establishing a method for the numerical simulation of carbon nanotube suspension forming processes. We focus our attention on spin coating processes, although we believe that the technique is general enough to be applied to other forming processes as well. Spin coating processes are used to apply very thin, more or less uniform, films onto flat substrates. In short, a drop of solution is placed in the center of the plate, which is then rotated at high speed. The centrifugal force makes the solution to spread on the flat substrate until the desired thickness is achieved on the film. The solvent is sometimes volatile in this kind of forming processes, an effect that is not considered in this work.

From the simulation point of view, spin coating processes involving Carbon nanotubes suspensions present some very important challenges. First of all, tracking the evolution of the free surface of the liquid, that undergoes very large deformations, remains a problem for traditional, Eulerian or Arbitrary Lagrangian Eulerian (ALE) Finite Element techniques [7], although some early references are available on the literature [9]. To this end, Lagrangian frameworks, in which the nodes of the simulation move with the material velocity, seem to ba an appropriate choice for the simulation. The family of meshless methods [2] [17] [24] seem to be an appealing choice, since they do not rely on a mesh (and consequently mesh distortion does not affect the accuracy of the results). In this work we employ a particular meshless method known as the Natural Element method and the method was proven to be capable of solving numerical problems that involve large distortion [18] [12].

The other remaining challenge is the constitutive modelling of CNT suspensions. In a first attempt, functionalized CNTs are considered as rigid fibres with very large aspect ratio (between $10^{3}-10^{4}$, possibly up to $10^{6}$, but typically in the order of hundreds after dispersion), whose orientation is governed by the flow of the solvent, which is Newtonian in this study. A quadratic closure relation is then applied to the preaveraged kinetic theory model. At the macroscopic scale, the flow kinematics of the suspension 


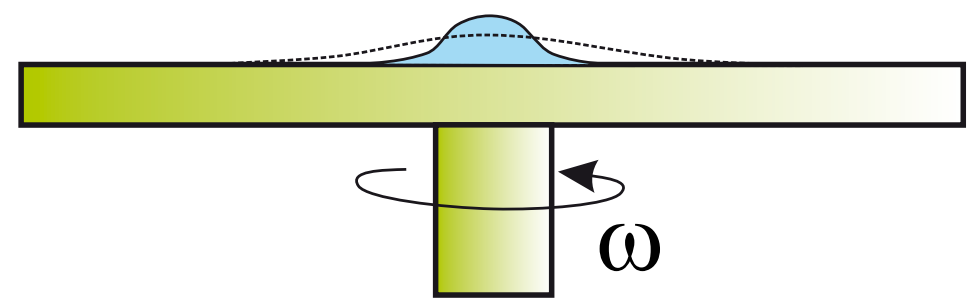

Fig. 1 Schematic representation of spin coating processes.

is governed by the momentum and mass conservation equations, which are further coupled with the micro-scale orientation of the CNTs. The CNT orientation and the suspension kinematics are updated at each time step as discussed in Section 6.

Some experimental measurements have been performed on the rheology of the CNTs suspensions, in order to characterize its behaviour. The CNTs modelled in this paper were chemically treated such that they did not aggregate and no mesostructures were observed optically.

The paper includes a brief description of the Natural Element Method (NEM) as well as the main assumptions involved the numerical modelling of spin coating. Some numerical examples are given to illustrate the potential of the proposed process simulation technique.

\section{Spin coating processes}

As mentioned before, spin coating processes consist of making a drop of liquid spread on a surface by means of centrifugal acceleration. This acceleration is achieved using a disk that rotates at a high angular velocity, $\omega$, see Fig. 1.

The difficulties in the numerical simulation of spin coating processes arise from (1) the tremendous deformation of the domain (in spin coating, the boundary can deform from a liquid droplet to thin films with a thickness of $30-50 \mu \mathrm{m}$ ) and (2) the multiscale nature of the problem.

In this work, the rotating plate is assumed to be perfectly horizontal, so that there is no radial component of gravity. Following [9], we assume that Coriolis forces are negligible, compared to centrifugal forces. To model the geometry of the drop, axial symmetry around the rotation axis is also assumed. A system of reference that rotates with the disc was used in describing the equations of motion.

\section{Numerical modelling of the spin coating process}

Carbon Nanotube (CNT) suspensions are modelled as short fibre suspensions described by the following equations (see [19] and references therein)

- The balance of momentum equations, where we only consider the centrifugal forces as in the treatment of [9]

$$
\operatorname{Div} \boldsymbol{\sigma}=-\rho \boldsymbol{\omega} \times(\boldsymbol{\omega} \times \boldsymbol{r})=\boldsymbol{f}_{r}
$$

where $\boldsymbol{\sigma}$ is the stress tensor, $\rho$ represents the density, $\times$ denotes the tensor product, and $\boldsymbol{\omega}$ the rotation speed of the spin coater as schematically drawn in Fig. 1. 
- The incompressibility condition

$$
\operatorname{Div} \boldsymbol{v}=0
$$

where $\boldsymbol{v}$ represents the velocity field.

- The constitutive equation, with a quadratic closure relation for the fourth order orientation tensor and other simplifying assumptions, results

$$
\boldsymbol{\sigma}=-p \boldsymbol{I}+2 \eta\left\{\boldsymbol{D}+N_{p} \operatorname{Tr}(\boldsymbol{a} \boldsymbol{D}) \boldsymbol{a}\right\}
$$

where $p$ denotes the pressure, $\boldsymbol{I}$ is the unit tensor, $\eta$ is the equivalent suspension viscosity, $\boldsymbol{D}$ is the strain rate tensor, $N_{p}$ a scalar parameter that depnds on both the tube concentration and its aspect ratio, and $\boldsymbol{a}$ is the second order orientation tensor defined by

$$
\boldsymbol{a}=\oint \boldsymbol{\rho} \otimes \rho \Psi(\rho) d \rho
$$

where $\rho$ is the unit vector defining the CNT axis direction, $\otimes$ denotes the dyadic product, and $\Psi(\boldsymbol{\rho})$ is the orientation distribution function, that should satisfy the normality condition

$$
\oint \Psi(\rho) d \rho=1
$$

If $\Psi(\boldsymbol{\rho})=\delta(\boldsymbol{\rho}-\hat{\boldsymbol{\rho}})$, with $\delta()$ the Dirac's function, all the orientation probability is concentrated in the direction defined by $\hat{\boldsymbol{\rho}}$, and the corresponding orientation tensor is $\hat{\boldsymbol{a}}=\hat{\boldsymbol{\rho}} \otimes \hat{\boldsymbol{\rho}}$. In this case the quadratic closure relation becomes exact. However, when the tubes are not perfectly aligned in a certain direction the quadratic closure is no more exact and it constitutes an approximation that is widely used in the context of short fibre suspensions, see [19] and references therein.

From a physical point of view, we can consider that the eigenvalues of the second order orientation tensor $(\boldsymbol{a})$ represent the probability of finding the CNT in the direction of the corresponding eigenvectors.

- With a quadratic closure relation the orientation equation is expressed as

$$
\frac{d \boldsymbol{a}}{d t}=\boldsymbol{\Omega} \boldsymbol{a}-\boldsymbol{a} \boldsymbol{\Omega}+k(\boldsymbol{D} \boldsymbol{a}+\boldsymbol{a} \boldsymbol{D}-2 \operatorname{Tr}(\boldsymbol{a D}) \boldsymbol{a})-6 D_{r}\left(\boldsymbol{a}-\frac{\boldsymbol{I}}{3}\right)
$$

$\boldsymbol{D}$ and $\boldsymbol{\Omega}$ are the symmetric and skew-symmetric components of $\operatorname{Grad} \boldsymbol{v}, k$ is a constant that depends on the nanotube aspect ratio $r$ (fibre length to fibre diameter ratio): $k=\left(r^{2}-1\right) /\left(r^{2}+1\right)$ with $k \approx 1$ given the high aspect ratio of the CNTs. $D_{r}$ is a rotary diffusion coefficient accounting for randomising events such as Brownian motion and fibre-fibre interaction in the suspension.

The flow model is defined in the volume occupied by the fluid at time $t, \Omega_{f}(t)$. On its boundary, $\Gamma_{f}(t) \equiv \partial \Omega_{f}(t)$ either the velocity or the traction is imposed:

$$
\boldsymbol{v}\left(\boldsymbol{x} \in \Gamma_{1}\right)=\boldsymbol{v}_{g}
$$

or

$$
\boldsymbol{\sigma} \boldsymbol{n}\left(\boldsymbol{x} \in \Gamma_{2}\right)=\boldsymbol{F}_{g}
$$

with $\Gamma_{1} \cup \Gamma_{2}=\Gamma_{f}(t), \Gamma_{1} \cap \Gamma_{2}=\emptyset$, and where $\boldsymbol{n}(\boldsymbol{x})$ is the unit outwards vector, defined on the boundary at the point $\boldsymbol{x}$. 
In terms of the initial condition, the CNTs are assumed to be isotropically orientated (i.e., $\boldsymbol{a}=\boldsymbol{I} / 3$ ). This model is solved on the domain defined by the nodes as they evolve. More details can be found at [19] and references therein.

In this modelling, the following form of the orientation vector is used for describing CNTs suspended within the solvent:

$$
\boldsymbol{\rho}=\left(\begin{array}{l}
r \\
\theta \\
z
\end{array}\right)
$$

with $\theta \in[0,2 \pi[$.

The velocity field is expressed in cylindrical coordinates, after the imposition of axial symmetry:

$$
\boldsymbol{v}=\left(\begin{array}{c}
v_{r} \\
\omega \cdot r \\
v_{z}
\end{array}\right)
$$

where $\omega$, as mentioned before, represents the angular velocity of the spin coater. If, following [9], we assume a system of reference rotating with the disk, we arrive at

$$
\boldsymbol{v}=\left(\begin{array}{c}
v_{r} \\
0 \\
v_{z}
\end{array}\right)
$$

In this case, tensors $\boldsymbol{D}$ and $\boldsymbol{\Omega}$ come from an axisymmetric flow, so their expression in cylindrical coordinates, as mentioned before, gives:

$$
\boldsymbol{D}=\left(\begin{array}{ccc}
\frac{\partial v_{r}}{\partial r} & \frac{1}{2}\left(\frac{1}{r} \frac{\partial v_{r}}{\partial \theta}+\frac{\partial v_{\theta}}{\partial r}-\frac{v_{\theta}}{r}\right) & \frac{1}{2}\left(\frac{\partial v_{r}}{\partial z}+\frac{\partial v_{z}}{\partial r}\right) \\
\frac{1}{2}\left(\frac{1}{r} \frac{\partial v_{r}}{\partial \theta}+\frac{\partial v_{\theta}}{\partial r}-\frac{v_{\theta}}{r}\right) & \frac{1}{r} \frac{\partial v_{\theta}}{\partial \theta}+\frac{v_{r}}{r} & \frac{1}{2}\left(\frac{1}{r} \frac{\partial v_{z}}{\partial \theta}+\frac{\partial v_{\theta}}{\partial z}\right) \\
\frac{1}{2}\left(\frac{\partial v_{r}}{\partial z}+\frac{\partial v_{z}}{\partial r}\right) & \frac{1}{2}\left(\frac{1}{r} \frac{\partial v_{z}}{\partial \theta}+\frac{\partial v_{\theta}}{\partial z}\right) & \frac{\partial v_{z}}{\partial z}
\end{array}\right)
$$

and, provided that the flow is axially symmetric $\left(v_{\theta}=0, v_{r}=v_{r}(r, z), v_{z}=v_{z}(r, z)\right)$, and by combining the above equation with Eq. (11) $\boldsymbol{D}$ reduces to

$$
\boldsymbol{D}=\left(\begin{array}{ccc}
\frac{\partial v_{r}}{\partial r} & 0 & \frac{1}{2}\left(\frac{\partial v_{r}}{\partial z}+\frac{\partial v_{z}}{\partial r}\right) \\
0 & \frac{v_{r}}{r} & 0 \\
\frac{1}{2}\left(\frac{\partial v_{r}}{\partial z}+\frac{\partial v_{z}}{\partial r}\right) & 0 & \frac{\partial v_{z}}{\partial z}
\end{array}\right)
$$

Similarly, the vorticity tensor takes the following form:

$$
\boldsymbol{\Omega}=\left(\begin{array}{ccc}
0 & 0 & \frac{1}{2}\left(\frac{\partial v_{r}}{\partial z}-\frac{\partial v_{z}}{\partial r}\right) \\
0 & 0 & 0 \\
-\frac{1}{2}\left(\frac{\partial v_{r}}{\partial z}-\frac{\partial v_{z}}{\partial r}\right) & 0 & 0
\end{array}\right)
$$

An isotropic orientation is defined in this model by the uniform distibution

$$
\psi(\rho)=\frac{1}{4 \pi}
$$

giving rise to an orientation tensor, for isotropic distributions,

$$
\boldsymbol{a}=\frac{1}{3} \boldsymbol{I}
$$

These conditions are imposed at the initial time step only. 


\section{Rheology of functionalized CNTs suspensions}

A Fokker-Planck (FP) based orientation model was used to describe the steady shear rheological responses of the chemically treated CNT suspensions. Fig. 2(a) shows the model fitting of surface-treated CNT suspensions with three different weight concentrations $(0.05 \%, 0.2 \%$ and $0.33 \%)$. Taking the $0.3 \%$ CNT suspension as an example, the evolution of the apparent viscosity $\eta_{a}$ in the orientation model is controlled by the values of $D_{r}$ and $N_{p}$ and the best constant- $D_{r}$ fit was obtained for $D_{r}=0.005 \mathrm{~s}^{-1}$ and $N_{p}=7$. In Fig. 2(b), different values of $D_{r}$ were used to illustrate the sensitivity of the model on the fitting parameter $D_{r}$. In general, there is a good agreement between $\eta_{a}$ predicted by the model and the experimental data, but for high $\dot{\gamma}_{R}$, the predicted $\eta_{a}$ is slightly higher than the experimental value. For instance, at shear rate $\dot{\gamma}_{R}=60 \mathrm{~s}^{-1}, \eta_{a}$ of the $0.3 \%$ suspension was found experimentally to be $10.5 \mathrm{~Pa} \cdot \mathrm{s}$, but the orientation model predicted a viscosity of $11.3 \mathrm{~Pa} \cdot s$ (with a constant $D_{r}=0.005 \mathrm{~s}^{-1}$ ) and this gives an error of about $8 \%$. Although there is an error of a few percents in predicting high-shear-rate data, the use of a constant $D_{r}$ should be sufficient to provide a reasonably good estimation of $\eta_{a}$ for general engineering problems, and this also saves the effort in determining the exact relationship between $D_{r}$ and $\dot{\gamma}_{R}$. However, in cases where a more accurate description of high-shear viscosity is needed, the dependence of $D_{r}$ on should be carefully evaluated (see for example, [10] [16]). Moreover, our recent modelling work suggested that the FP based orientation model cannot satisfactorily describe the shear-thinning characteristics for untreated CNT suspensions where optically resolvable CNT aggregates are present (see for example [21]). For untreated CNT suspensions, a new aggregation/orientation model should be used and in that model, diffusion coefficient depends not only on the orientation of CNTs, but also on the entanglement state of CNTs. Detailed model formulation and fittings for both treated and untreated CNT suspensions can be found in the article entitled "The Rheological Modelling of Carbon Nanotube (CNT) Suspensions" in this special issue.

\section{Basics of the Natural Element (NE) Method}

As mentioned in the introductory section, we have chosen to employ a meshless method, the Natural Element Method, to perform the simulation, mainly because it allows for a Lagrangian description of the flow kinematics, convenient for solving free-surface flow problems with large surface deformation. The basics of the method are described next.

\subsection{Natural Neighbour interpolation}

The vast majority of meshless methods are based on the use of scattered data approximation techniques to construct the approximating spaces of the Galerkin method. These techniques must have, of course, low sensitivity to mesh distortion, as opposed to Finite Element (FE) methods. Among these techniques, the Natural Element Method employs any instance of Natural Neighbour interpolation [23] [14] to construct trial and test functions. Prior to the introduction of these interpolation techniques, it is necessary to define some basic concepts.

The model will be constructed upon a cloud of points with no connectivity established a priori among them. We will call this cloud of points $N=\left\{n_{1}, n_{2}, \ldots, n_{M}\right\} \subset$ 


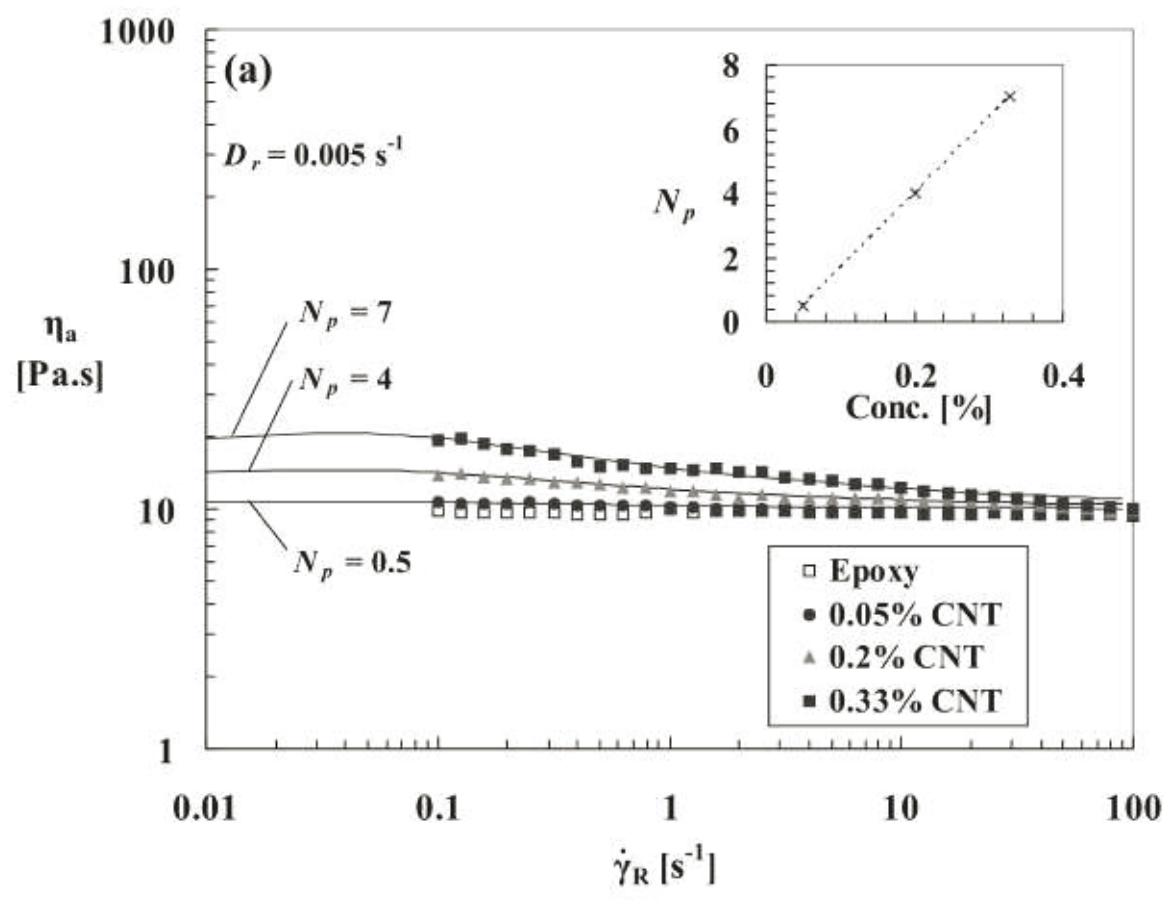

(a)

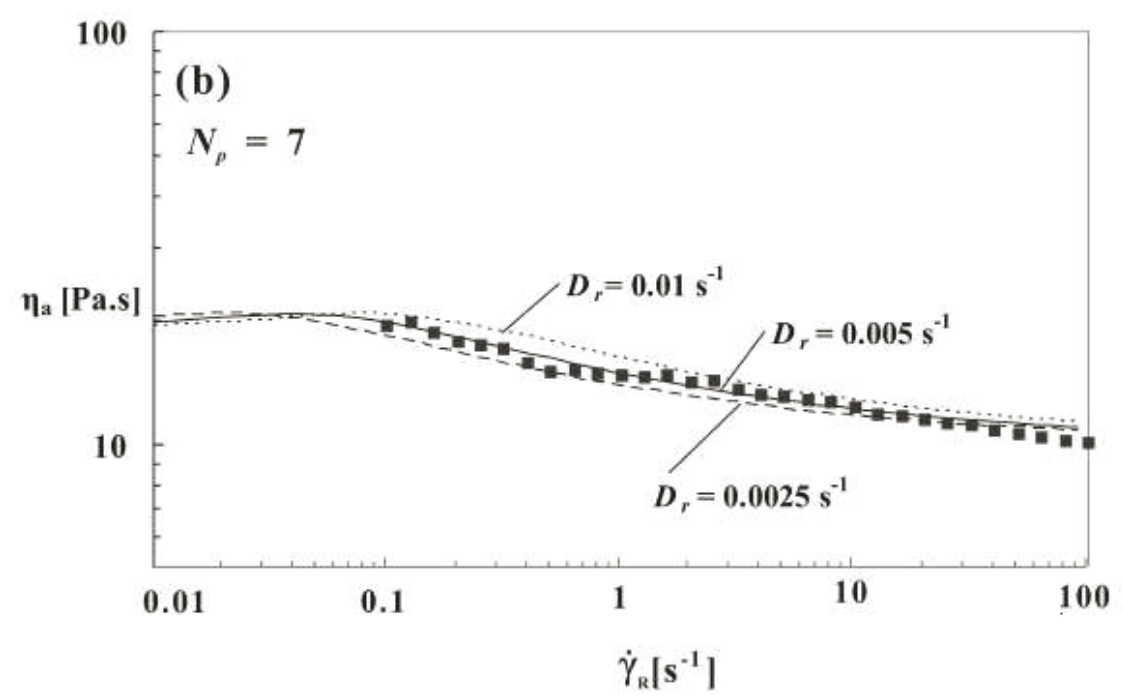

(b)

Fig. 2 (a) Orientation model fitting of $0.05 \%, 0.2 \%$ and $0.33 \%$ surface-treated CNTs suspended in epoxy resin. $N_{p}$ and $D_{r}$ are the fitting parameters. (b) Sensitivity of the model to $D_{r}$ given the experimental data of $0.33 \%$ treated CNT suspension and $N_{p}=7$.

$\mathbb{R}^{d}$, and there is a unique decomposition of the space into regions such that each point within these regions is closer to the node with which the region is associated than to any other in the cloud. This kind of space decomposition is called a Voronoi diagram of the cloud of points and each Voronoi cell is formally defined as (see figure 3):

$$
T_{I}=\left\{\boldsymbol{x} \in \mathbb{R}^{d}: d\left(\boldsymbol{x}, \boldsymbol{x}_{I}\right)<d\left(\boldsymbol{x}, \boldsymbol{x}_{J}\right) \forall J \neq I\right\},
$$

where $d(\cdot, \cdot)$ is the Euclidean distance function.

The dual structure of the Voronoi diagram is the Delaunay triangulation, obtained by connecting nodes that share a common $(d-1)$-dimensional facet. While the Voronoi 

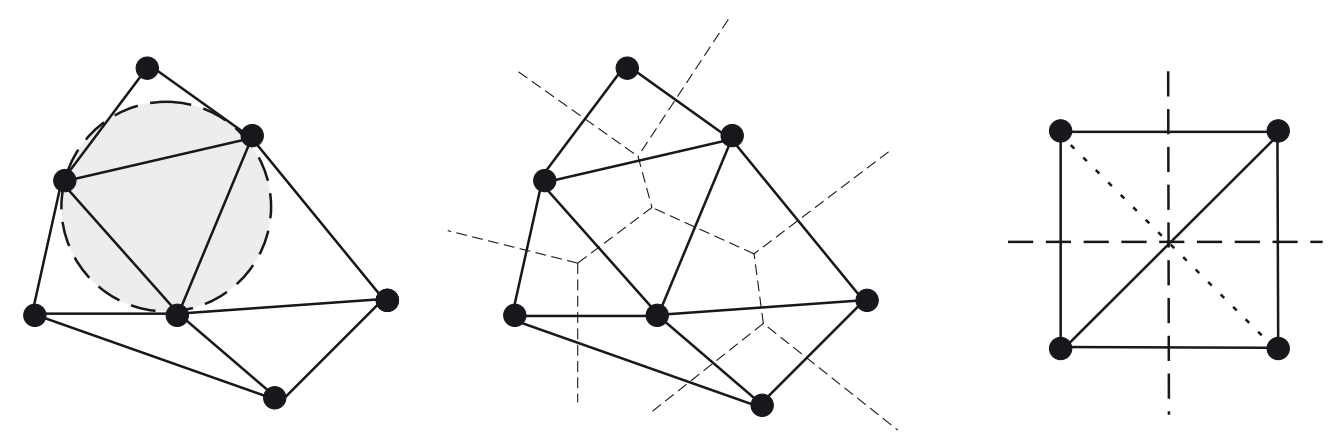

Fig. 3 Delaunay triangulation and Voronoi diagram of a cloud of points.

structure is unique, the Delaunay triangulation is not, there exist some degenerate cases in which there are two or more possible Delaunay triangulations (consider, for example, the case of triangulating a square in 2D, as depicted in Fig. 3 (right)). Another way to define the Delaunay triangulation of a set of nodes is by invoking the empty circumcircle property, which means that no node of the cloud lies within the circle covering a Delaunay triangle. Two nodes sharing a facet of their Voronoi cell are called natural neighbours and hence the name of the technique.

In order to define the natural neighbour co-ordinates it is necessary to introduce some additional concepts. The second-order Voronoi diagram of the cloud is defined as

$$
T_{I J}=\left\{\boldsymbol{x} \in \mathbb{R}^{d}: d\left(\boldsymbol{x}, \boldsymbol{x}_{I}\right)<d\left(\boldsymbol{x}, \boldsymbol{x}_{J}\right)<d\left(\boldsymbol{x}, \boldsymbol{x}_{K}\right) \forall J \neq I \neq K\right\} .
$$

The most common natural neighbour interpolation method, is the Sibson interpolant [22] [23]. Consider the introduction of the point $\boldsymbol{x}$ in the cloud of nodes. Due to this introduction, the Voronoi diagram will be altered, affecting the Voronoi cells of the natural neighbours of $\boldsymbol{x}$. Sibson [22] defined the natural neighbour coordinates of a point $\boldsymbol{x}$ with respect to one of its neighbours $I$ as the ratio of the cell $T_{I}$ that is transferred to $T_{x}$ when adding $\boldsymbol{x}$ to the initial cloud of points to the total volume of $T_{x}$. In other words, if $\kappa(\boldsymbol{x})$ and $\kappa_{I}(\boldsymbol{x})$ are the Lebesgue measures of $T_{x}$ and $T_{x I}$ respectively, the natural neighbour coordinates of $\boldsymbol{x}$ with respect to the node $I$ is defined as

$$
\phi_{I}(\boldsymbol{x})=\frac{\kappa_{I}(\boldsymbol{x})}{\kappa(\boldsymbol{x})} .
$$

In Fig. 4 the shape function associated to node 1 may be expressed as

$$
\phi_{1}(\boldsymbol{x})=\frac{A_{a b f e}}{A_{a b c d}} .
$$

It is straightforward to prove that NE shape functions (see Fig. 5) form a partition of unity [1], as well as some other properties like positivity (i.e., $0 \leq \phi_{I}(\boldsymbol{x}) \leq 1 \forall I, \forall \boldsymbol{x}$ ) and strict interpolation:

$$
\phi_{I}\left(\boldsymbol{x}_{J}\right)=\delta_{I J}
$$

Sibson interpolants have some remarkable properties that help to construct the trial and test functional spaces of the Galerkin method (see [24], [14]).

Besides properties like continuity and smoothness (everywhere except at the nodes for Sibson interpolants), Sibson interpolants posses linear completeness (i.e., exact reproduction of a linear field) and it is noteworthy that they are able to exactly interpolate prescribed essential (Dirichlet) boundary conditions, a condition usually not fulfilled by other meshless methods. 


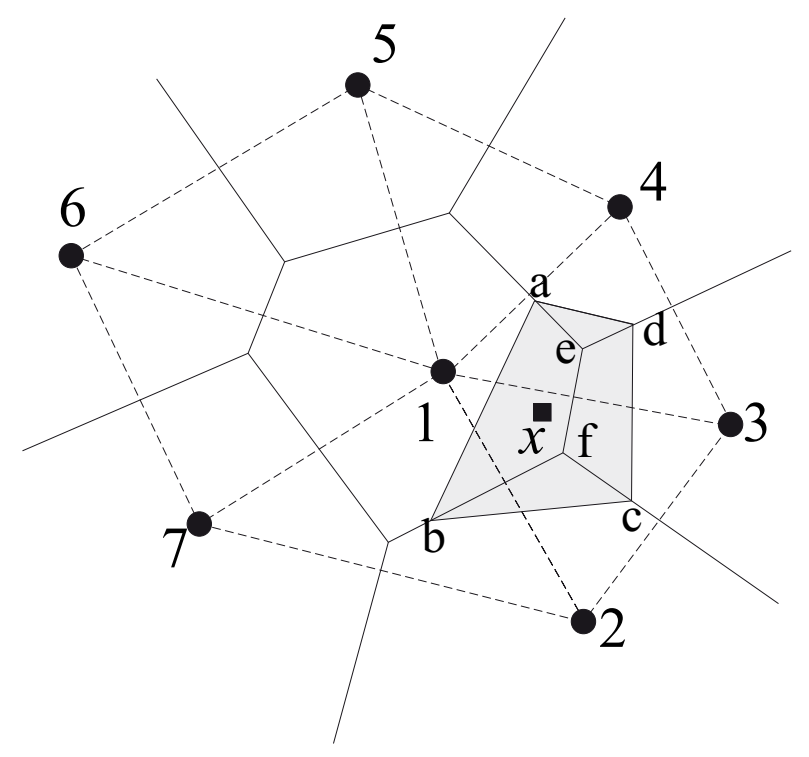

Fig. 4 Definition of the Natural Neighbour coordinates of a point $\boldsymbol{x}$.
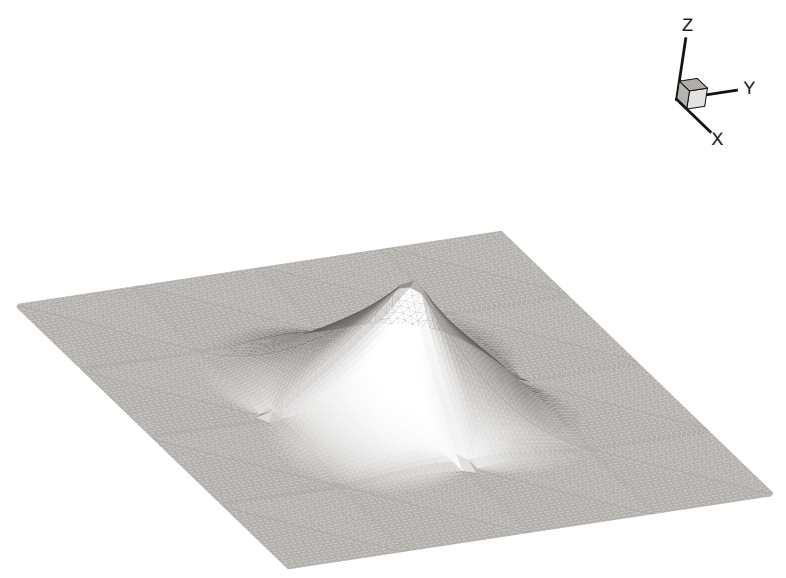

Fig. 5 Typical function $\phi(\boldsymbol{x})$.

\subsection{The $\alpha$-shapes-based Natural Element Method}

The identification of the free surface in an updated Lagrangian flow simulation deserves some comments. Using meshless methods, in which models are constructed by a set of nodes only, boundary tracking can be performed by employing different strategies. In particular, we have employed shape constructors to perform this task. Shape constructors are geometrical entities that transform finite point sets into a multiply connected shape in general. In particular, we employ $\alpha$-shapes [8]. $\alpha$-shapes define a one-parameter family of shapes $\mathcal{S}_{\alpha}$ (being $\alpha$ the parameter), ranging from the "coarsest" to the "finest" level of detail. $\alpha$ can be seen, precisely, as a measure of this level of detail.

Details about the formal definition of the family of $\alpha$-shapes can be found in [8]. In brief, the use of $\alpha$-shapes to define the boundary of the domain relies in the choice of the level of detail needed to represent the domain, which is always an analyst's decision. It is obvious that the minimum nodal spacing parameter, say $h$, should be chosen so as to reproduce at least that level of detail $\alpha$. 


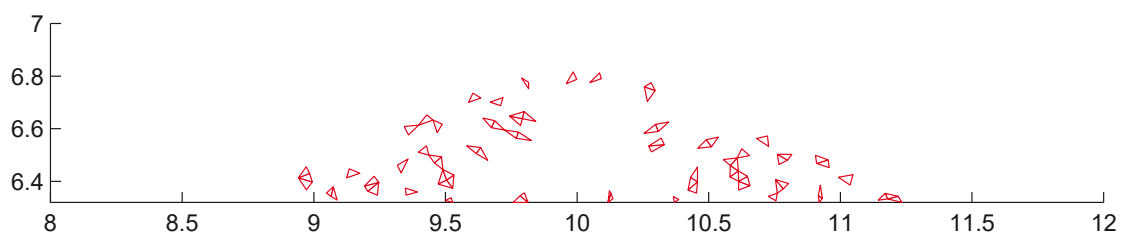

(a)

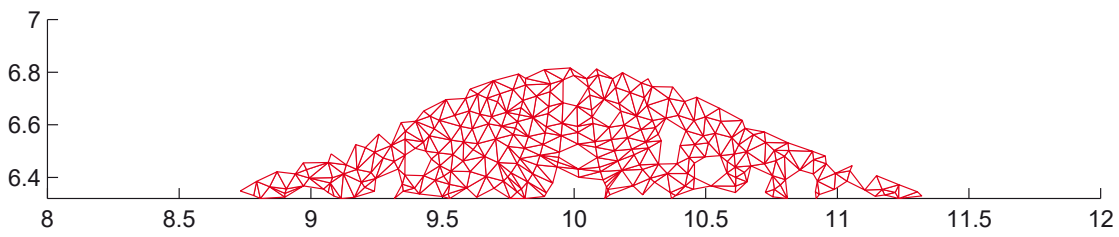

(b)

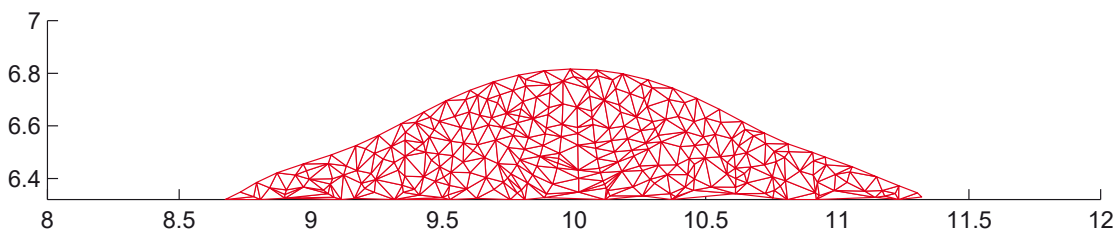

(c)

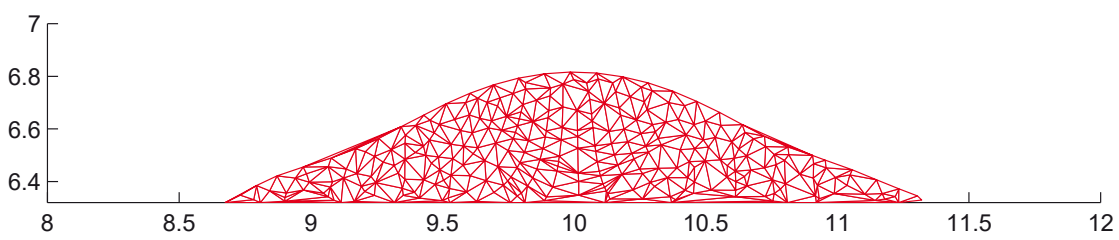

(d)

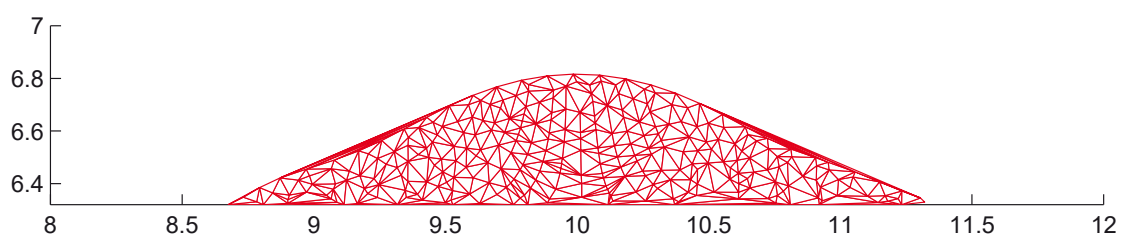

(e)

Fig. 6 Evolution of the family of $\alpha$-shapes of a cloud of points representing drop impacting on a flat surface. Shapes $\mathcal{S}_{0.03}$ (a), $\mathcal{S}_{0.05}$ (b), $\mathcal{S}_{0.5}$ (c), $\mathcal{S}_{2.0}$ (d), $\mathcal{S}_{100.0} \sim \mathcal{S}_{\infty}$ (e) are depicted.

$\alpha$-shapes provide a means to eliminate from the triangulation those triangles or tetrahedra whose size is bigger than the specified level of detail. This criterion is very simple: just perform a filtration to eliminate those triangles (tetrahedra) whose circumradius is bigger than the level of detail, $\alpha$.

In Fig. 6 an example of the previously presented theory is presented. It represents some instances of the finite set of shapes for a cloud in a intermediate step of the simulation of drop impacting onto a flat surface. The experimental solution of this problem, together with some snapshots of its numerical simulation, are shown in Fig. 7 and 8.

Note that the key question in using $\alpha$-shapes is not to find the precise value of $\alpha$ for a given configuration of the nodal cloud. Instead, we must set the problems in terms of what level of detail we are interested in taking into account for a particular geometry. 

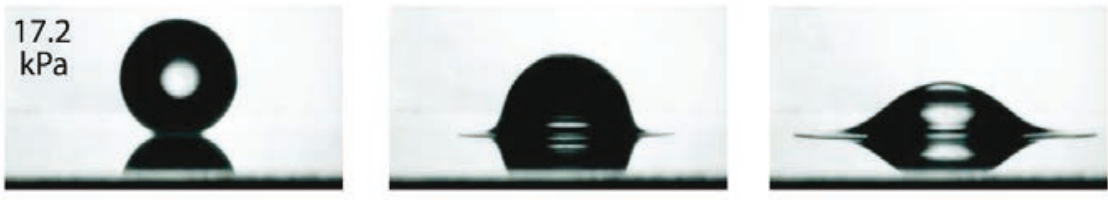

Fig. 7 Sequence of the drop deformation under very low ambient pressure. Photos courtesy of Lei Xu and Sidney Nagel.
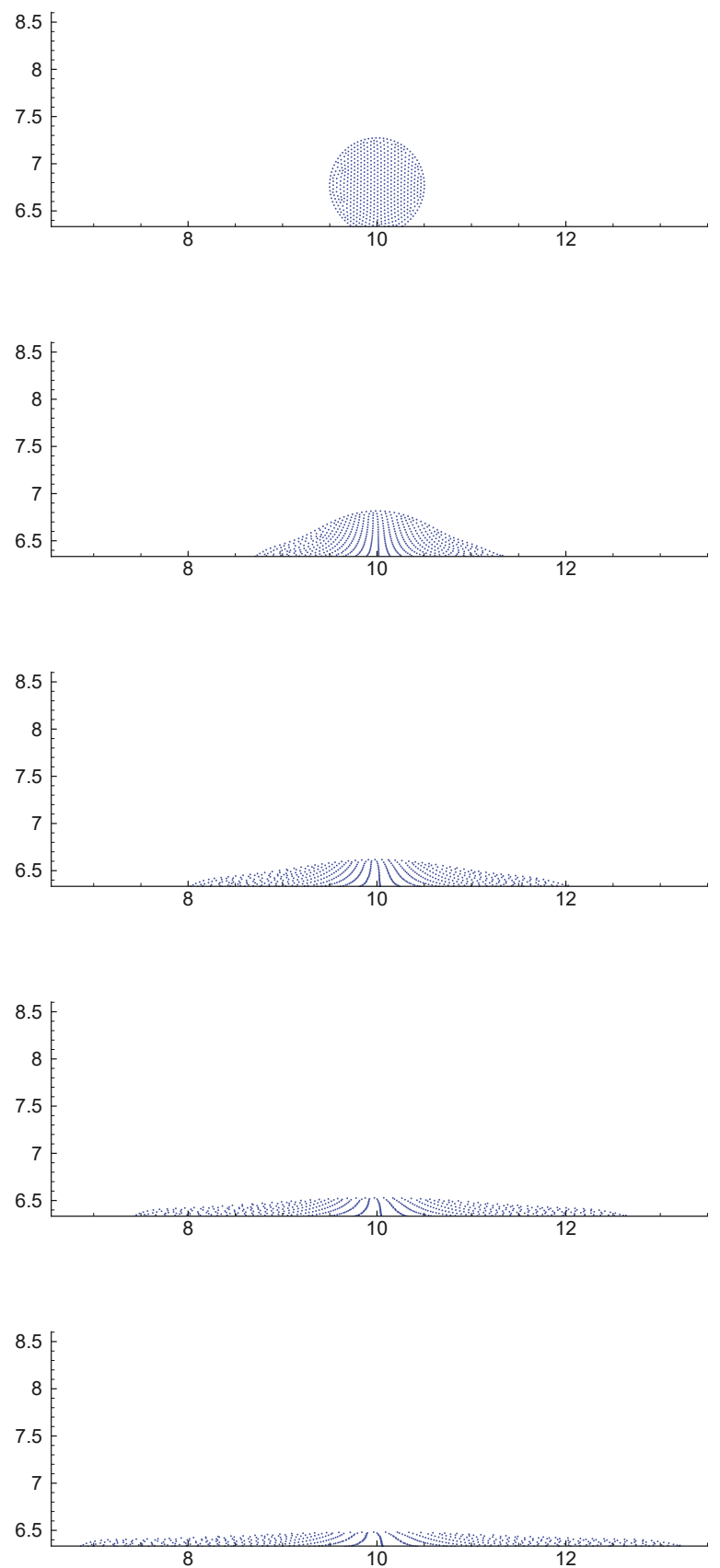

Fig. 8 Evolution of the free surface in the drop impact. 
The use of $\alpha$-shapes, however, has another relevant influence in the Natural Element Method. As demonstrated in [4], the construction of natural neighbour interpolation on an $\alpha$-shape of the domain alters the distance measure. Natural neighbour interpolation is performed on the basis of Voronoi diagrams, which employ euclidean distance measure in their most general form. This leads to some lack of interpolation along nonconvex boundaries. This interpolation is recovered if we construct the natural neighbour interpolants over an $\alpha$-shape of the domain.

Thus, the use of $\alpha$-shapes in the construction of updated Lagrangian simulations of fluid flow provides an appealing way to track the boundary of domain while ensuring appropriate interpolation of essential boundary conditions, that can be imposed directly in the discrete system of equations, as in the Finite Element Method.

\section{Discrete model}

A mixed $\mathcal{C}^{0}-\mathcal{C}^{-1} \mathrm{NE}$ interpolation has been applied to discretize the strong form of the problem introduced before. More details on the application of mixed NE interpolations in incompressible and nearly incompressible media can be found in [5].

In the formulation here presented, a $\mathcal{C}^{0}$ interpolation scheme - smooth everywhere except at the nodes - has been chosen in the velocity field approximation, whereas a discontinuous $\mathcal{C}^{-1}$ interpolation has been used in the pressure approximation:

$$
\begin{gathered}
\boldsymbol{v}^{h}(\boldsymbol{x})=\sum_{I=1}^{n} \phi_{I}(\boldsymbol{x}) \boldsymbol{v}_{I} \\
p^{h}(\boldsymbol{x})=\sum_{I=1}^{n} \psi_{I}(\boldsymbol{x}) p_{I}=\sum_{I=1}^{n} \frac{1}{n} p_{I}
\end{gathered}
$$

where $\boldsymbol{v}_{I}$ and $p_{I}$ represent the nodal velocities and pressures, respectively, and $n$ is the number of natural neighbours of the point $\boldsymbol{x}$ under consideration.

This kind of approximation does not verify the LBB condition. However, it has been shown that its behaviour is very similar to that of the bilinear velocity-constant pressure finite element (see [13]). No spurious modes or locking have been observed in all the simulations computed in this paper.

Thus, with the fluid domain $\Omega_{f}(t)$ extracted at time $t$ from the cloud of nodes by using the $\alpha$-shape technique, as described in the previous section, and the velocity and pressure natural element interpolation defined by Eqs. (22) and (23), we can proceed to a standard discretisation of the mixed variational formulation of the flow equations

$$
\begin{gathered}
\int_{\Omega_{f}(t)} \boldsymbol{\sigma}: \boldsymbol{D}^{*} d \Omega=\int_{\Omega_{f}(t)} \boldsymbol{f}_{r} \boldsymbol{v}^{*} d \Omega \\
\int_{\Omega_{f}(t)} \operatorname{Div} \boldsymbol{v} p^{*} d \Omega=0
\end{gathered}
$$

with

$$
\boldsymbol{\sigma}=-p \boldsymbol{I}+2 \eta\left\{\boldsymbol{D}+N_{p} \operatorname{Tr}(\boldsymbol{a} \boldsymbol{D}) \boldsymbol{a}\right\}
$$

where a null traction is assumed on the flow front and a prescribed velocity is enforced on the other part of the fluid domain boundary. Essential boundary conditions involve setting the velocity of the nodes in contact with the rotating plate to zero. 
The orientation equation is solved at each time increment. With the flow kinematics known at time $t, \boldsymbol{v}^{t}(\boldsymbol{x})$, the position of nodes can be updated at the same time that the fibres orientation evolution, given by Eq. (6) is computed by using the method of characteristics. The simplest explicit updating consists in writing

$$
\boldsymbol{x}_{I}^{t+\Delta t}=\boldsymbol{x}_{I}^{t}+\boldsymbol{v}_{I}^{t} \Delta t, \quad \forall I
$$

and

$$
\begin{gathered}
\boldsymbol{a}_{I}^{t+\Delta t}=\boldsymbol{a}_{I}^{t}+\left\{\boldsymbol{\Omega}_{I}^{t} \boldsymbol{a}_{I}^{t}-\boldsymbol{a}_{I}^{t} \boldsymbol{\Omega}_{I}^{t}+k \boldsymbol{D}_{I}^{t} \boldsymbol{a}_{I}^{t}+k \boldsymbol{a}_{I}^{t} \boldsymbol{D}_{I}^{t}-\right. \\
\left.-2 k \operatorname{Tr}\left(\boldsymbol{a}_{I}^{t} \boldsymbol{D}_{I}^{t}\right) \boldsymbol{a}_{I}^{t}-6 D_{r}\left(\boldsymbol{a}_{I}^{t}-\frac{\boldsymbol{I}}{3}\right)\right\} \Delta t, \quad \forall I
\end{gathered}
$$

where $\boldsymbol{D}_{I}^{t}$ and $\boldsymbol{\Omega}_{I}^{t}$ are the symmetric and skew-symmetric components of the gradient of velocity tensor, at time $t$ in the node $\boldsymbol{x}_{I}$, respectively.

Finally, in the kinematics resolution stage, the fibre orientation described by the second order orientation tensor $\boldsymbol{a}$ is assumed to be known at the nodes at present time step, $\boldsymbol{a}_{I}^{t}$. The value of $\boldsymbol{a}$ at the integration points used to evaluate Eqs. (24) and (25) is computed by using the natural element interpolation

$$
\boldsymbol{a}^{t}(\boldsymbol{x})=\sum_{I=1}^{I=n} \phi_{I}(\boldsymbol{x}) \boldsymbol{a}_{I}^{t}
$$

The only difficulty in applying Eq. (28) to update the orientation is related to the non-derivability of the natural element shape functions at their definition nodes. Thus, we can evaluate the velocity gradient tensor from the expression

$$
\boldsymbol{v}_{, k}^{t}(\boldsymbol{x})=\sum_{I=1}^{I=n} \phi_{I_{, k}}(\boldsymbol{x}) \boldsymbol{v}_{I}^{t}
$$

where the subscripts $k$ denotes the spatial derivative with respect to the $k$-coordinate. The velocity gradient can be computed everywhere except at the nodes, since $\phi_{I_{k}}\left(\boldsymbol{x}_{I}\right)$ is not defined. A possible solution for this relies in the use of Stabilised Conforming Nodal integration techniques [3][11] or to use some kind of projection from integration points and then averaging [25] [6].

\section{Numerical results}

The previously presented model was applied to the simulation of spinning a drop of CNT suspension, with the assumption that the initial shape is Gaussian. The model was composed of 977 nodes under axisymmetric assumptions for the flow (but not for the orientation field, which is three-dimensional). Non-slip boundary conditions were assumed at the horizontal plane, whereas symmetry boundary conditions are assumed at the axis of symmetry. Some snapshots for the simulated evolution of the velocity field for different time steps are shown in Fig. 9.

The results on the orientation field for the carbon nanotubes are shown in Figs. 10-11. It can be noticed how CNTs tend to adopt an horizontal orientation in the $r-z$ plane, due to the gradient of velocities in this plane, very close to pure shear due to the non-slip boundary conditions. On the $\theta-z$ plane, however, and by virtue of the diverging velocity field, CNTs tend to align to the $\theta$ direction, although the ellipses are not as distorted as those on the $r-z$ plane and the degree of alignment is less significant. 


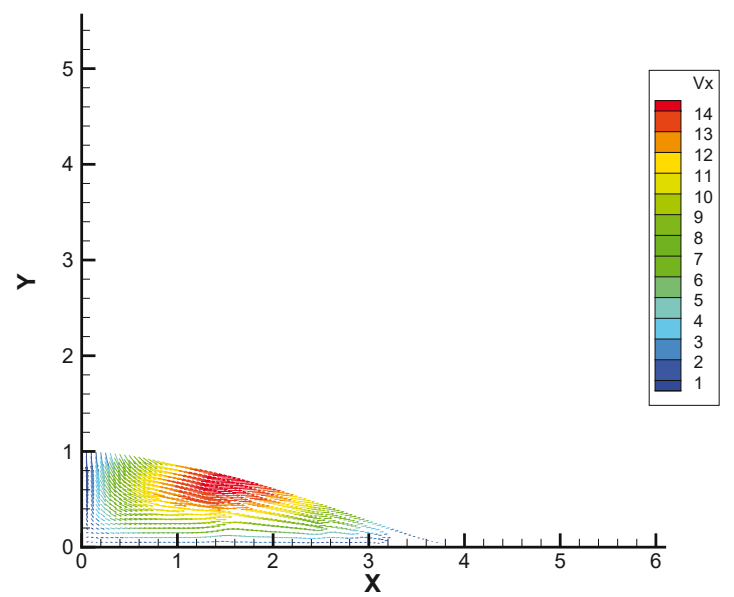

(a)

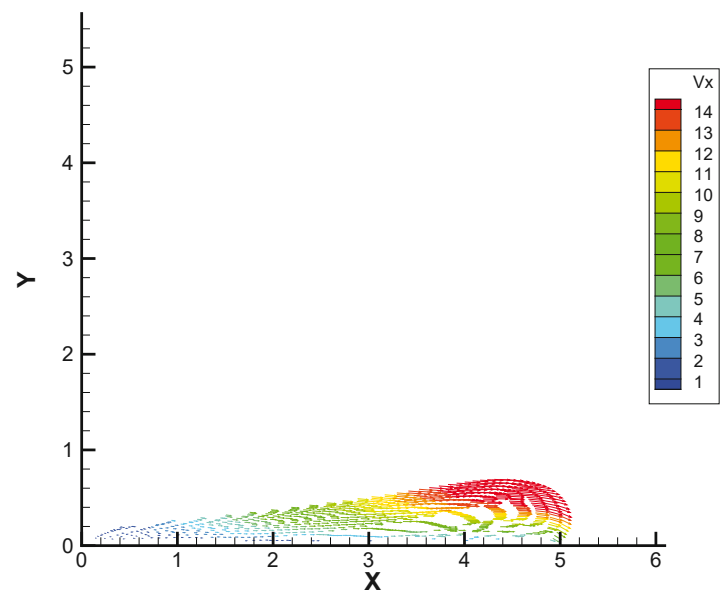

(c)

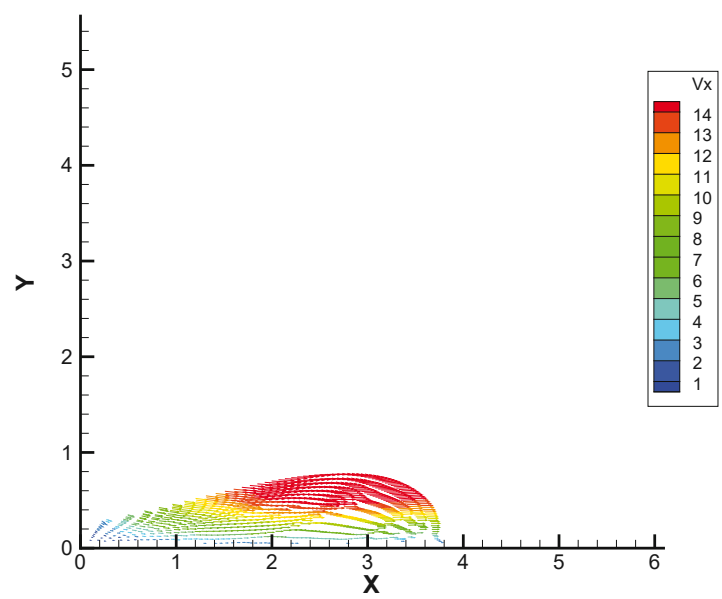

(b)

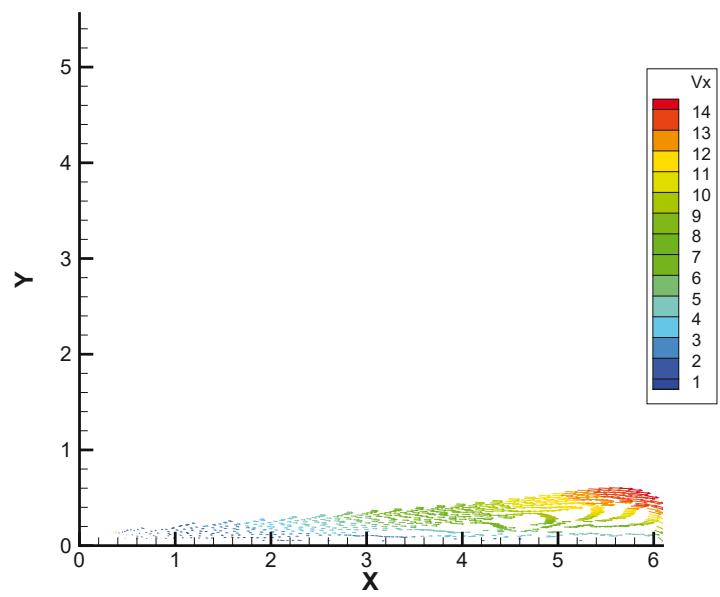

(d)

Fig. 9 Snapshots of the velocity field at $1^{\text {st }}, 10^{t h}, 20^{t h}$ and $30^{t h}$ time steps. $\Delta t=0.01 \mathrm{~s}$.

\section{Conclusions}

A model has been presented for the numerical simulation of spin coating of carbon nanotubes suspensions. The model is composed of a meshless (natural element) strategy for the flow that allows for a proper description of the free-surface flow and a micromacro description of the nanotube scale that allows for a suitable description of CNT orientation field.

The use of a meshless strategy in an updated Lagrangian framework, combined with an $\alpha$-shape approach, to describe the large motion of the free surface seems to be an appealing choice, due to its simplicity. In this way since nodes move with material velocity, the orientation field can be stored at the nodes and be updated easily by a simple algorithm based on the method of characteristics.

Other possibilities, such as the kinetic description derived from the Fokker-Planck equation or its equivalent stochastic (Itô) counterpart could also be applied in this 


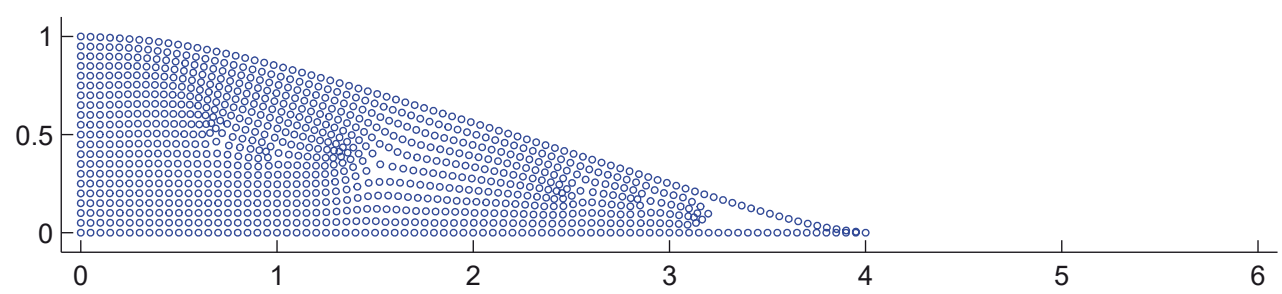

(a)

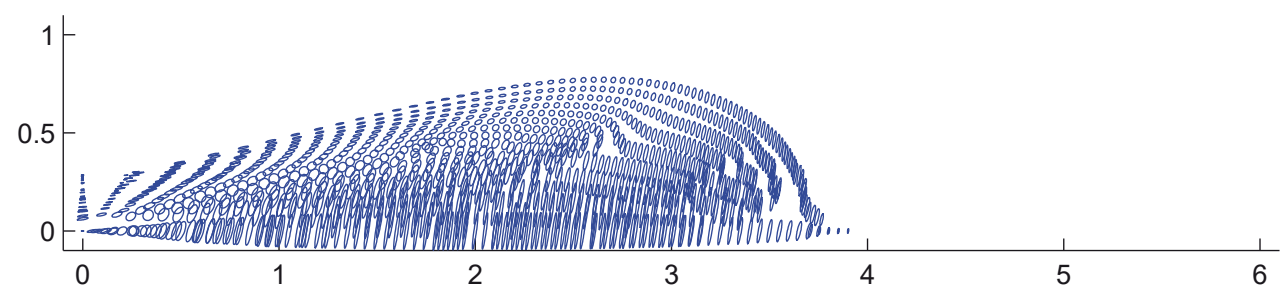

(b)

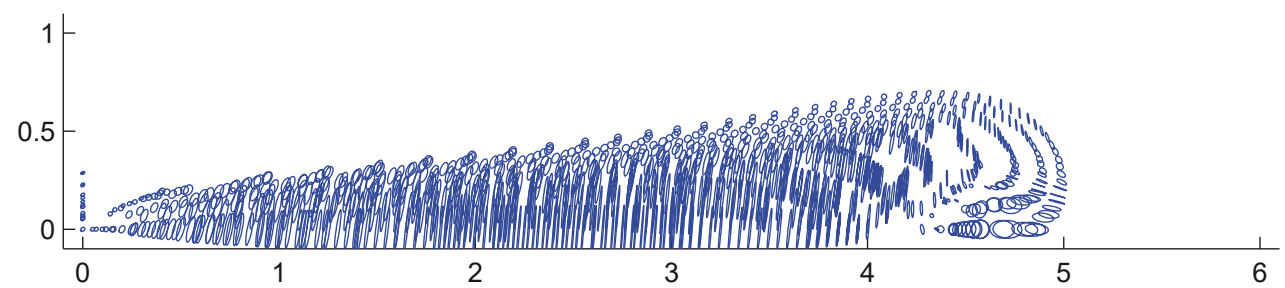

(c)

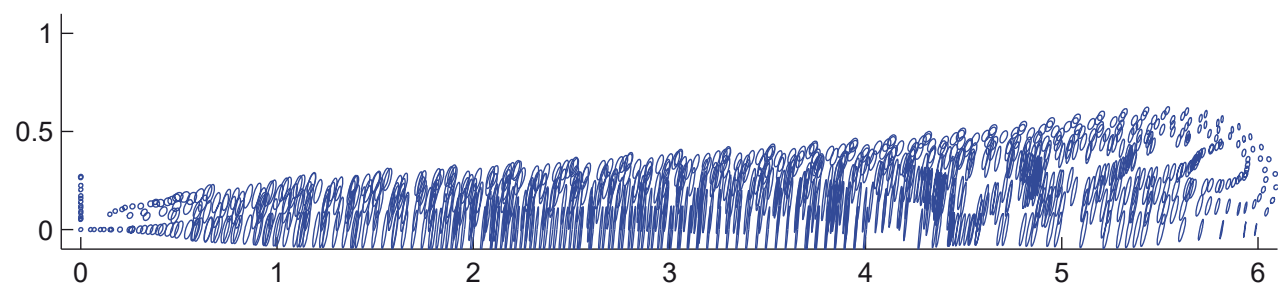

(d)

Fig. 10 Orientation field for the CNT suspension at some intermediate steps of the simulation $(r-z$ orientation field $) .1^{s t}, 10^{t h}, 20^{t h}$ and $30^{t h}$ time steps.

same framework. This remains an important part of ongoing research and the findings will be reported in a future paper.

\section{References}

1. Babuška, I., Melenk, J.M.: The partition of unity method. International Journal for Numerical Methods in Engineering 40, 727-758 (1997)

2. Belytschko, T., Lu, Y.Y., Gu, L.: Element-Free Galerkin Methods. International Journal for Numerical Methods in Engineering 37, 229-256 (1994)

3. Chen, J.S., Wu, C.T., Yoon, S., You, Y.: A stabilized conforming nodal integration for galerkin mesh-free methods. International Journal for Numerical Methods in Engineering 50, 435-466 (2001)

4. Cueto, E., Doblaré, M., Gracia, L.: Imposing essential boundary conditions in the Natural Element Method by means of density-scaled $\alpha$-shapes. International Journal for Numerical Methods in Engineering 49-4, 519-546 (2000)

5. Cueto, E., Martínez, M.A., Doblaré, M.: El método de los Elementos Naturales en Elasticidad compresible y cuasi-incompresible. Boletín Técnico del Instituto de Materiales y Modelos Estructurales. Universidad Central de Venezuela. (In spanish) 39 (2001) 


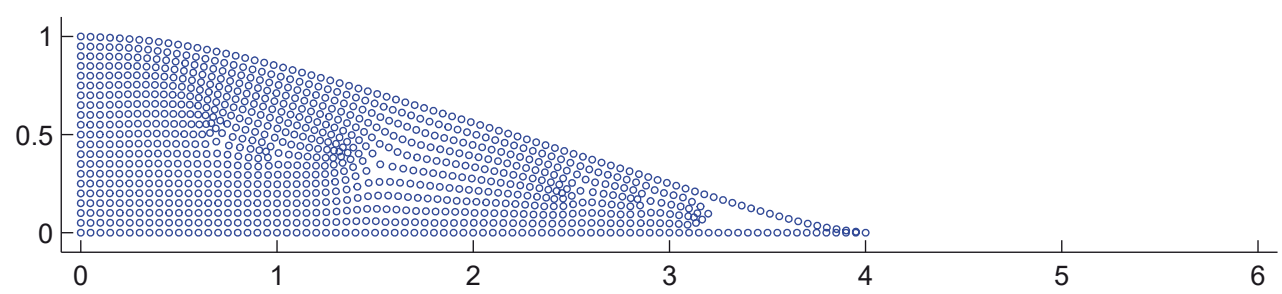

(a)

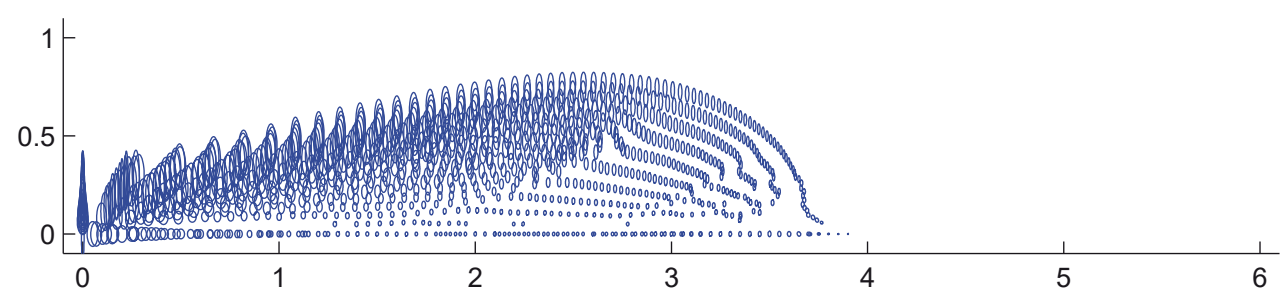

(b)

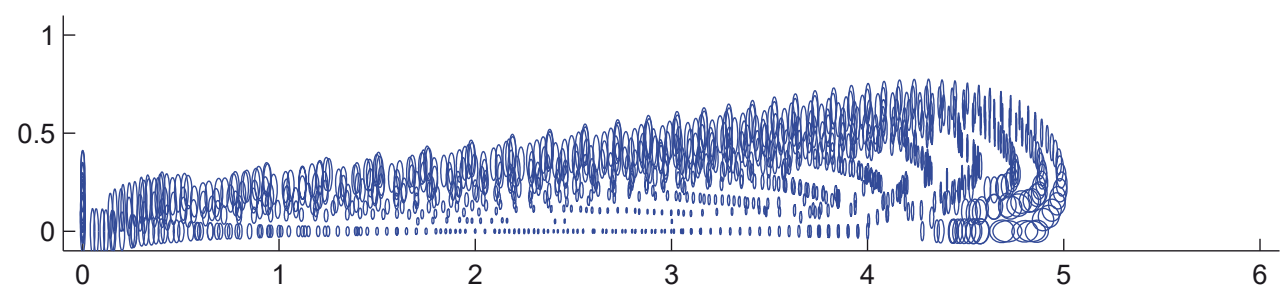

(c)

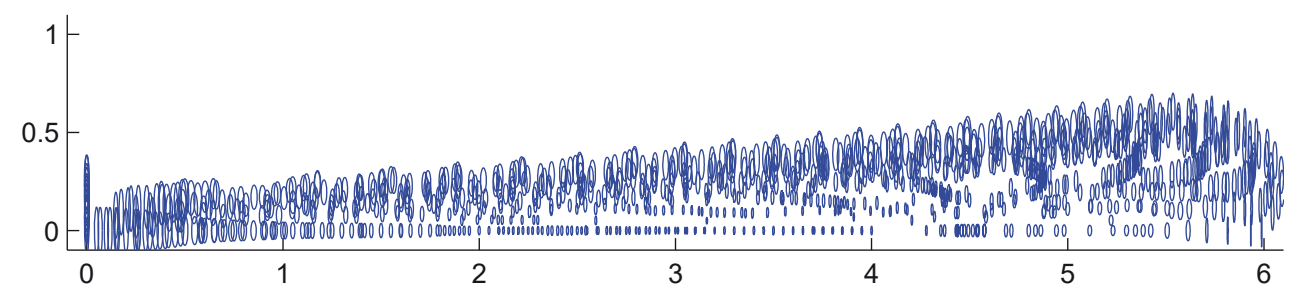

(d)

Fig. 11 Orientation field for the CNT suspension at some intermediate steps of the simulation $(r-\theta$ plane $) .1^{\text {st }}, 10^{t h}, 20^{t h}$ and $30^{t h}$ time steps.

6. Cueto, E., Sukumar, N., Calvo, B., Martínez, M.A., Cegoñino, J., Doblaré, M.: Overview and recent advances in Natural Neighbour Galerkin methods. Archives of Computational Methods in Engineering 10(4), 307-384 (2003)

7. Donea, J.: Arbitrary Lagrangian-Eulerian finite element methods. In: T. Belytschko, T.J.R. Hughes (eds.) Computer methods for transient analyses, pp. 474-516. ElsevierAmsterdam (1983)

8. Edelsbrunner, H., Mücke, E.: Three dimensional alpha shapes. ACM Transactions on Graphics 13, 43-72 (1994)

9. Emslie, A.G., Bonner, F.T., Peck, L.G.: Flow of a viscous liquid on a rotating shell. Journal of Applied Physics 29(5), 858-863 (1958)

10. Folgar, F., III, C.L.T.: Orientation behaviour of fibers in concentrated suspensions. J. Reinf. Plast. Compos. 3, 98-119 (1984)

11. Gonzalez, D., Cueto, E., Martinez, M.A., Doblare, M.: Numerical integration in Natural Neighbour Galerkin methods. International Journal for Numerical Methods in Engineering 60(12), 2077-2104 (2004)

12. González, D., Cueto, E., Chinesta, F., Doblaré, M.: A natural element updated Lagrangian strategy for free-surface fluid dynamics. Journal of Computational Physics 223(1), 127$150(2007)$ 
13. González, D., Cueto, E., Doblaré, M.: Volumetric locking in Natural Neighbour Galerkin methods. International Journal for Numerical Methods in Engineering 61(4), 611-632 (2004)

14. Hiyoshi, H., Sugihara, K.: Two generalizations of an interpolant based on Voronoi diagrams. International Journal of Shape Modeling 5(2), 219-231 (1999)

15. Iijima, S.: Single-shell carbon nanotubes of 1-nm diameter. Nature 363, 603-605 (1991)

16. Larson, R.G.: The Structure and Rheology of Complex Fluids. Oxford University Press (1999)

17. Liu, W.K., Jun, S., Li, S., Adee, J., Belytschko, T.: Reproducing kernel particle methods. International Journal for Numerical Methods in Engineering 38, 1655-1679 (1995)

18. Martinez, M.A., Cueto, E., Alfaro, I., Doblare, M., Chinesta, F.: Updated Lagrangian free surface flow simulations with Natural Neighbour Galerkin methods. International Journal for Numerical Methods in Engineering 60(13), 2105-2129 (2004)

19. Martínez, M.A., Cueto, E., Doblaré, M., Chinesta, F.: Fixed mesh and meshfree techniques in the numerical simulation of injection processes involving short fiber suspensions. Journal of Non-Newtonian Fluid Mechanics 115, 51-78 (2003)

20. Postma, H.W.C., Teepen, T., Yao, Z., Grifoni, M., Dekker, C.: Carbon nanotube singleelectron transistors at room temperature. Science 293, 5527 (2001)

21. Rahatekar, S.S., Koziol, K.K.K., Butler, S.A., Elliott, J.A., Mackley, M., Windle, A.H.: Optical microstructure and viscosity enhancement for an epoxy resin matrix containing multi-wall carbon nanotubesorientation behaviour of fibers in concentrated suspensions. Journal of Rheology 50, 599-610 (2006)

22. Sibson, R.: A Vector Identity for the Dirichlet Tesselation. Mathematical Proceedings of the Cambridge Philosophical Society 87, 151-155 (1980)

23. Sibson, R.: A brief description of natural neighbour interpolation. In: Interpreting Multivariate Data. V. Barnett (Editor), pp. 21-36. John Wiley (1981)

24. Sukumar, N., Moran, B., Belytschko, T.: The Natural Element Method in Solid Mechanics. International Journal for Numerical Methods in Engineering 43(5), 839-887 (1998)

25. Zienkiewicz, O.C., Zhu, J.Z.: The Superconvergent Patch Recovery and a posteriori error estimates. Part I: the recovery technique. International Journal forn Numerical Methods in Engineering 33, 1331-1364 (1992) 